\title{
Magnetocaloric Effect in Materials with the First Order Transitions — Direct Measurements
}

 \\ ${ }^{a}$ Institute of Physics ASCR, v.v.i., Na Slovance 2, 18221 Praha 8, Czech Republic \\ ${ }^{b}$ Charles University in Prague, Faculty of Mathematics and Physics \\ Ke Karlovu 3, 12116 Praha 2, Czech Republic
}

\begin{abstract}
The results of direct measurements of the magneto-caloric characteristics $\Delta T_{\mathrm{ad}}$ and the indirect determination of $\Delta S_{\mathrm{m}}$ of pure $\mathrm{Gd}$ around the Curie temperature and of the FeMnP $\mathrm{P}_{0.45} \mathrm{As}_{0.55}$ and $\mathrm{Ni}_{45} \mathrm{Co}_{5} \mathrm{Mn}_{31} \mathrm{Ga}_{19}$ intermetallics in the vicinity of the first order magnetic and structural phase transitions are presented and discussed. A pronounced temperature and field dependence of the first order transitions in the studied materials manifests restrictions applying these materials in magnetic refrigeration techniques. An effective use of the Ericsson and the Brayton refrigeration cycles with $\mathrm{FeMnP}_{0.45} \mathrm{As}_{0.55}$ in the role of a refrigerant is discussed.
\end{abstract}

PACS numbers: $75.30 . \mathrm{Sg}, 75.30 . \mathrm{Kz}, 75.50 . \mathrm{Cc}$

\section{Introduction}

The magneto-caloric effect (MCE) is characterized either by the adiabatic change of temperature $\Delta T_{\text {ad }}$ or by the isothermal change of magnetic entropy $\Delta S_{\mathrm{m}}$, both being caused by a change of magnetic field. After the first use of the magneto-caloric properties of $\mathrm{Gd}$ in a refrigeration process [1], physics of this effect has been intensively studied $[2,3]$ and a large effort is paid now to find materials with the giant MCE around room temperature [4-8]. The very promising MCE characteristics have been discovered in systems based on the rare-earth elements, like $\mathrm{Gd}_{5}(\mathrm{Si}, \mathrm{Ge})_{4}[4,5]$, or in intermetallic compounds with the transition metal elements, like FeMnPAs [6], MnAs [7], and $\mathrm{Ni}_{2} \mathrm{MnGa}$ [8].

The adiabatic $\Delta T_{\mathrm{ad}}$ can be measured directly and $\Delta S_{\mathrm{m}}$ is determined indirectly using the Maxwell relation and the measured magnetization isotherms $M(H)_{T}$ :

$$
\Delta S_{\mathrm{m}}=\int(\mathrm{d} M / \mathrm{d} T)_{H} \mathrm{~d} H .
$$

However, a relevance of the Maxwell equation to a description of MCE in materials with the first order magnetic phase transitions (FOMPT) has been widely discussed in the last years $[2,3,5]$. We present the MCE characteristics of pure Gd that exhibits the second order magnetic transition and of the polycrystalline FeMnP $\mathrm{P}_{0.45} \mathrm{As}_{0.55}$ and $\mathrm{Ni}_{45} \mathrm{Co}_{5} \mathrm{Mn}_{31} \mathrm{Ga}_{19}$ intermetallics with FOMPT and structural phase transitions to assess the applicability of these materials in refrigeration techniques.

\section{Experimental}

The direct measurements of $\Delta T_{\mathrm{ad}}$ have been carried out in a simple cryostat and the Halbach type of a permanent magnet with transversal magnetic field $1 \mathrm{~T}$. The

\footnotetext{
* corresponding author; e-mail: kamarad@fzu.cz
}

arrangement allows a rapid change of magnetic field by a rate of $3 \mathrm{~T} / \mathrm{s}$ that can be treated as adiabatic. The induced $\Delta T$ is recorded by the shielded miniature $\mathrm{Ni}-\mathrm{NiCr}$ thermocouples with time steps of $300 \mathrm{~ms}$. Together with the following isofield cooling (or heating) of a sample by a heat transfer between the sample and a massive sample holder, the experimental arrangement can be considered as a model of the Brayton cycle of refrigeration. The magnetization isotherms used for the determination of $\Delta S_{\mathrm{m}}$ were measured in the SQUID magnetometer (QUANTUM Design Co.) at field up to $5 \mathrm{~T}$.

\section{Results and discussion}

MCE in Gd was observed in a wide temperature range $(\approx 45 \mathrm{~K})$ around the Curie temperature $T_{\mathrm{C}}=293 \mathrm{~K} \mathrm{[1].}$ We have observed the maximum values of $\Delta T_{\mathrm{ad}}=2.6 \mathrm{~K}$ (direct measurement) and $\Delta S_{\mathrm{m}}=2.8 \mathrm{~J} /(\mathrm{kg} \mathrm{K})($ magnetization measurement) induced by $\Delta H=1 \mathrm{~T}$ at temperature $295 \mathrm{~K}$. These values agree well with relevant room temperature values of $\Delta T_{\mathrm{ad}}=5.8 \mathrm{~K}$ and $\Delta S_{\mathrm{m}}=5.5 \mathrm{~J} /(\mathrm{kg} \mathrm{K})$ presented in Ref. [3] for $\Delta H=2 \mathrm{~T}$.

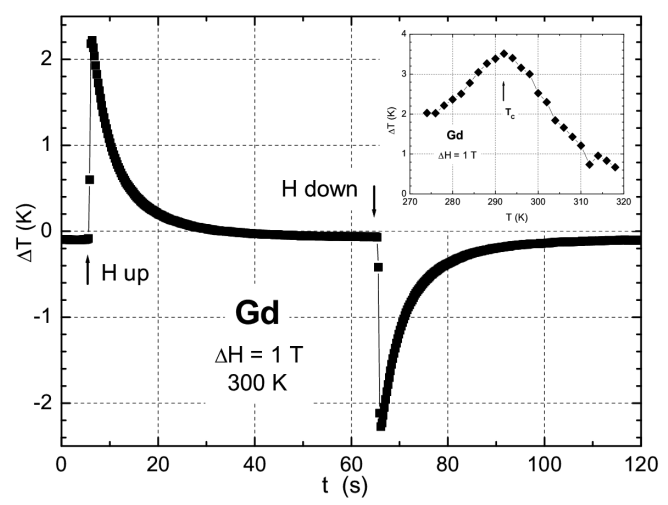

Fig. 1. Time dependence of $\Delta T$ induced in $\mathrm{Gd}$ by $\Delta H=1 \mathrm{~T}$ at the temperature of $300 \mathrm{~K}$. Inset: temperature dependence of $\left|\Delta T_{\mathrm{ad}}\right|$ in a wide range around $T_{\mathrm{C}}$. 


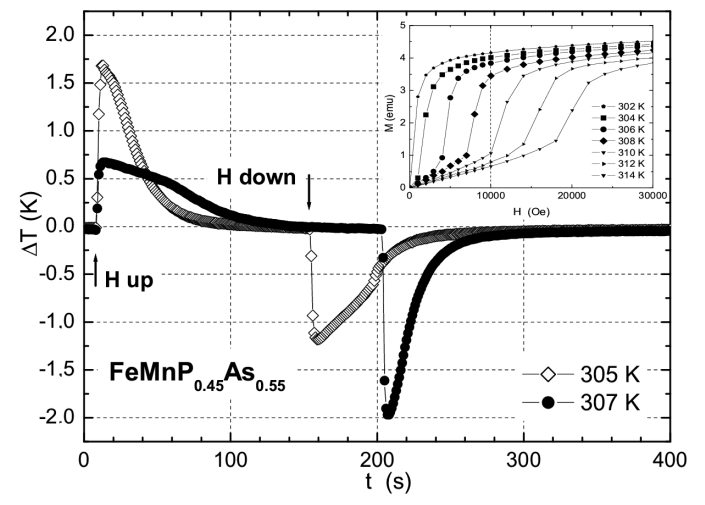

Fig. 2. $\Delta T$ induced in $\mathrm{FeMnP}_{0.45} \mathrm{As}_{0.55}$ by $\Delta H=1 \mathrm{~T}$ at temperatures of $305 \mathrm{~K}$ and $307 \mathrm{~K}$. Inset: magnetization isotherms at temperatures around FOMPT.

However, the values of $\Delta T_{\text {ad }}$ calculated from magnetization measurements (see inset in Fig. 1) are of about $20 \%$ higher than those determined from the direct MCE measurements. The exponential decrease (or increase) of the sample temperature after the adiabatic field change presented in Fig. 1 excellently obeys the known Newton cooling law.

FeMnP $_{0.45} \mathrm{As}_{0.55}$ exhibits FOMPT at the critical temperature $T_{\mathrm{C}}=306 \mathrm{~K}$, without any hysteresis. The critical field $H_{\mathrm{c}}$ of the meta-magnetic transition above $T_{\mathrm{C}}$ increases rapidly with increasing temperature $\left(\mathrm{d} H_{\mathrm{c}} / \mathrm{d} T=\right.$ $+2 \mathrm{kOe} / \mathrm{K})$, see inset in Fig. 2 . In the case of $\Delta H=1 \mathrm{~T}$, $\mathrm{MCE}$ can be observed in a very narrow temperature range around $T_{\mathrm{C}}$ only and a relatively high value of $\Delta S_{\mathrm{m}}=10.5 \mathrm{~J} /(\mathrm{kg} \mathrm{K})$ at $T=306 \mathrm{~K}$ determined by the Maxwell relation is misleading in this case. Due to the high value of $\mathrm{d} H_{\mathrm{c}} / \mathrm{d} T$, the adiabatic magnetization curves are substantially different with respect to the presented magnetization isotherms. A heating (or cooling) of the sample by MCE $\left(\Delta T_{\mathrm{ad}}=2 \mathrm{~K}\right)$ leads to the increase (or decrease) of $H_{\mathrm{c}}$ by about $0.4 \mathrm{~T}$. Hence, the saturated magnetization cannot be reached in our experiment by increasing field to $1 \mathrm{~T}$ at a temperature close to the top of the MCE temperature range. On the other hand, the sample partially remains in ferromagnetic state after the adiabatic release of the field at a temperature close to the bottom of this temperature range. As can be seen in Fig. 2, the maximum value of $\left|\Delta T_{\mathrm{ad}}\right|=2 \mathrm{~K}$ is not reached in these cases. The following isofield cooling (or heating) of the sample cannot be described by the Newton law and the relevant curves indicate an additional isofield heating (or cooling) of the sample.

The shape memory effects in materials of the $\mathrm{Ni}_{2} \mathrm{MnGa}$ type are caused by the first order structural (martensiteaustenite) transition that is sensitive extremely to the magnetic field. In the case of $\mathrm{Ni}_{45} \mathrm{Co}_{5} \mathrm{Mn}_{31} \mathrm{Ga}_{19}$, the transition into austenite at $T_{\mathrm{A}}=345 \mathrm{~K}$ is accompanied by a substantial increase of the magnetization (see inset in Fig. 3) and hence, a pronounced negative MCE has been predicted using relation (1). However, the neg- ative $\mathrm{MCE}$ in $\mathrm{Ni}_{45} \mathrm{Co}_{5} \mathrm{Mn}_{31} \mathrm{Ga}_{19}$ is strongly suppressed by both, the dynamics and the hysteresis of the martensitic transition. $\Delta S_{\mathrm{m}}$ is small and the maximum value of $\left|\Delta T_{\text {ad }}\right|$ is less than $0.5 \mathrm{~K}$ for $\Delta H=1 \mathrm{~T}$. Moreover, the negative MCE is not reversible due to the hysteresis, see Fig. 3.

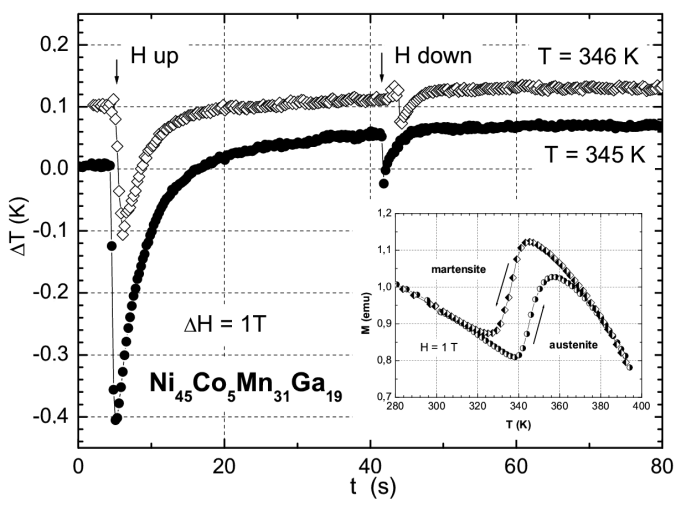

Fig. 3. Small $\Delta T$ induced by the negative MCE in $\mathrm{Ni}_{45} \mathrm{Co}_{5} \mathrm{Mn}_{31} \mathrm{Ga}_{19}$ at temperatures of $345 \mathrm{~K}$ and $346 \mathrm{~K}$. Inset: a hysteretic temperature dependence of $M(1 \mathrm{~T})$ nearby the martensitic transition.

The presented direct measurements of the MCE characteristics illustrate problems with a use of the materials as refrigerants in the Brayton cycle of refrigeration. The unavoidable difference between the adiabatic magnetization curves and the magnetization isotherms in materials with FOMPT plays the main role. From this point of view, the Ericsson cycle with isothermal changes of field and with following isofield cooling (or heating) of the magnetic refrigerant would be more effective.

\section{Acknowledgments}

The support of the Grant Agency of the Czech Republic (projects No. 202/09/0030 and 101/07/1173) is acknowledged.

\section{References}

[1] G.V. Brown, J. Appl. Phys. 47, 3673 (1976).

[2] N.A. de Oliveira, P.J. von Ranke, Phys. Rep. 489, 89 (2010).

[3] A.M. Tishin, Y.I. Spichkin, The Magnetocaloric Effect and its Applications, Institute of Physics Pub., Bristol 2003.

[4] V.K. Pecharsky, K.A. Gschneidner, Phys. Rev. Lett. 78, 4494 (1997).

[5] V.K. Pecharsky, K.A. Gschneidner, Ya. Mudryk, D. Paudyal, J. Magn. Magn. Mater. 321, 3541 (2009).

[6] O. Tegus, E. Brück, K.H.J. Buschow, F.R. de Boer, Nature 415, 150 (2002).

[7] H. Wada, Y. Tanabe, Appl. Phys. Lett. 79, 3302 (2001).

[8] F. Albertini, J. Kamarád, Z. Arnold, L. Pareti, E. Villa, L. Righi, J. Magn. Magn. Mater. 316, E35 (2007). 